Research Article

\title{
Digital Community Management Mobile Information System Based on Edge Computing
}

\author{
Junhua Xu \\ School of Economics and Information, Zhejiang Tongji Vocational College of Science and Technology, Hangzhou 3111231, \\ Zhejiang, China
}

Correspondence should be addressed to Junhua Xu; xujunhua@zjtongji.edu.cn

Received 14 May 2021; Revised 12 June 2021; Accepted 10 July 2021; Published 5 August 2021

Academic Editor: Sang-Bing Tsai

Copyright (C) 2021 Junhua Xu. This is an open access article distributed under the Creative Commons Attribution License, which permits unrestricted use, distribution, and reproduction in any medium, provided the original work is properly cited.

Edge computing refers to an open platform that integrates network, computing, storage, and application core capabilities on the side close to the source of things or data and provides nearest-end services nearby. "Digital Community" refers to the use of various information technologies and methods to integrate community resources and build a network platform for interactive exchanges and services between the government, property service agencies, residents, and various intermediary organizations within the community. In order to reduce the management tasks of community managers, reduce their community management burden, and improve the management efficiency of community managers, this article is based on the description of several keywords such as distributed edge computing, edge computing business usage scenarios, and digital community management. Edge computing technologies such as centralized traffic model and attribute-based trust evaluation avoid waste of network resources, reduce time delay and system energy consumption, and improve system operation efficiency. A digital community management mobile information management system is designed and developed. 102 owners of the community conducted visits and questionnaire surveys. After investigation and research, it was found that this system has good performance, meets the management needs of most communities, and can improve the management efficiency of community managers by $4 \%-6 \%$; it has also improved community residents' satisfaction with digital community management by $2-3 \%$.

\section{Introduction}

1.1. Background. Mobile information system refers to the comprehensive communication platform based on modern mobile communication technology and mobile Internet technology, through the use of handheld terminals, servers, personal computers, and other equipment to communicate and interact to provide a full range of management and services to the society. With the development of the global information economy, the digital construction of urban communities in many countries and regions has been explored in theory and practice for many years. In order to serve the country and improve international competitiveness, these cities are racing to introduce digital, networked, and information foundations. Facility construction policies and measures have gradually expanded from the initial e-government to the digital management of urban communities. It has been developed for a long time and has relatively rich experience. The digital management of communities has become an extremely important part of national governance and social development.

1.2. Significance. Mobile edge computing provides content providers and application developers with real-time radio access network (RAN) information, such as network load and user location information. This real-time network information can provide mobile users with context-aware services, thereby improving user experience and user service quality. Mobile edge computing adds corresponding responsibilities to the edge network, allows the edge network to perform tasks such as computing and services, and increases the management authority of the edge network to 
reduce the network delay and energy consumption of mobile users. Network operators allow third-party partners to process the edge of the wireless communication network. Therefore, for mobile users and enterprises, new applications and edge services will be deployed more quickly.

1.3. Related Work. Blockchain, as the backbone technology of the currently popular Bitcoin digital currency, has become a promising decentralized data management framework. Although blockchain has been widely adopted in many applications such as finance, healthcare, and logistics, its application in mobile services is still limited. This is due to the fact that blockchain users need to solve a preset proof-ofwork problem in order to add new data (i.e., blocks) to the blockchain. However, solving the workload proof consumes a lot of CPU time and energy resources and is not suitable for resource-constrained mobile devices. In order to promote blockchain applications in future mobile IoT systems, multiaccess mobile edge computing seems to be an auspicious solution to the proof-of-work problem of mobile users. Xiong et al. first introduced the new concept of edge computing for mobile blockchain. Then, they introduced an economic method for edge computing resource management. In addition, a prototype of a blockchain system with mobile edge computing functions is provided, and experimental results are provided to prove [1]. But they only made great contributions to medical research and did not conduct too much research on community management. Multiaccess edge computing (MEC) is an emerging ecosystem that aims to converge telecommunications and IT services, providing a cloud computing platform at the edge of the radio access network (RAN). MEC provides storage and computing resources at the edge, thereby reducing the waiting time of mobile end users and making more effective use of mobile backhaul and core networks. Taleb et al. introduced the MEC survey and focused on basic key supporting technologies. It has meticulously designed the MEC orchestration on the basis of considering a single service and the MEC platform network that supports mobility, which brings light to different orchestration deployment options. In addition, Taleb et al. analyzed the MEC reference architecture and main deployment scenarios, which provide multitenant support for application developers, content providers, and third parties. Finally, Taleb et al. outlined current standardization activities and further elaborated on the challenges of open research [2]. But they did not apply MEC technology to community services. The Multivillage Plan (MVS), which connects hundreds of villages and small towns to a large water distribution network, represents an emerging area of rural water supply in low-and middle-income countries. In this case, traditional rural water supply methods usually advocate community management, but the scale and complexity of MVS require alternative methods. Hutchings introduced three case studies of MVS from India, which focused on the role of the community in overall management. These illustrate the different mechanisms by which community management can or cannot be nested in the entire management system, as well as the different ways to promote community participation. The discussion provided an explanation for the differences between these case studies from the perspective of political economy, and from the perspective of public policy, Hutchings discussed how and why MVS can lead to the decline of community management in some cases [3]. But they only discussed why MVS would cause the decline of community management under certain circumstances and did not use the latest MEC technology to improve digital community management.

1.4. Innovation. Using edge computing to design a digital community management system can reduce system running time and response time and reduce server bandwidth and lower energy consumption. The responsible subject of the community management system uses various information platforms and uses big data technology to explore and analyze the frequency of "sensitive words" and "repetitive words" and actively understand the public sentiment of the community. Prevention of emergencies can guide community residents to actively participate in the evaluation of community services and management and fully express their individualized requirements for public products and public services. It is possible for public services and needs within a fixed period of time. We propose suggestions for improvement if there is a mismatch and then form a good multiparty interaction, information feedback, and measure correction mechanism. On the basis of solving information asymmetry, we realize the overall coordination of community service and management and system services, reduce community services, provide and use the intermediate cost, integrate service resources, improve use efficiency, accurately meet the all-round, personalized, and diversified needs of residents, and accurately, timely, and effectively monitor the type, scale, and quality of community service provision.

\section{Methods and Related Concepts}

2.1. Edge Computing. Cloud computing is a type of distributed computing, which refers to the process of decomposing huge data computing processing programs into countless small programs through the network "cloud" and then processing and analyzing these small programs through a system composed of multiple servers to obtain results and return to the user. Compared with edge computing, the huge computing power provided by cloud computing makes it meaningful to perform heavy work on huge and heavy data sets. Most of the time, the concentration of cloud computing is better than the decentralized nature of edge computing in terms of speed, cost, and scalability, but cloud computing also has many shortcomings. In order to solve the deficiencies of cloud computing, edge computing has begun to arouse widespread attention in academia and industry. There has not been a unified definition of edge computing since it was put forward. It is widely understood that edge computing is a kind of building edge cloud by integrating edge computing and storage facilities at the edge of the network to provide cloud services and an IT operating environment for edge applications. 
Mobile edge computing is one of the important components of $5 \mathrm{G}$ communication technology. From the installation location, MEC is generally at the edge of the mobile network and in the wireless access network.

Deployed in a location close to mobile users, MEC's main task is to reduce time delay, ensure efficient network operation and service delivery capabilities, and also achieve a better user experience. More software-defined networks are introduced in $5 \mathrm{G}$ communications, and IT virtualization technology is widely used in hardware infrastructure. MEC is a key technology and architecture for the evolution of $5 \mathrm{G}$ communications. It represents the transition from mobile broadband networks to software-defined networks. Latency, scalability, and throughput meet the demanding requirements of $5 \mathrm{G}$ networks. Figure 1 shows a simplified version of the MEC architecture. The MEC server is deployed next to the mobile base station to provide edge computing capabilities for terminal devices.

2.2. Edge Computing Business Scenarios. The main business scenarios of edge computing include but are not limited to the following three:

(1) Decentralized traffic model: a large number of sensors are connected to the edge of the network to generate huge uplink data and computing requests. The network traffic is developing from vertical to flat. The edge cloud makes local decisions at the source of the traffic and forwards it from east to west through clustering, reducing network bandwidth and central cloud pressure [4]

(2) Services that require stringent delay: in the fields of intelligent vehicle networking, unmanned driving, and intelligent manufacturing, most businesses require a delay of up to $10 \mathrm{~ms}$, which is an absolute delay-sensitive business. Through the nearby processing of the edge cloud, the network transmission delay can be greatly reduced to meet the needs of such applications [5]

(3) Data preprocessing business [6]: smart IoT devices continuously generate massive amounts of streaming data, which can perform preprocessing such as data filtering and desensitization when the data flows through the edge cloud, which can not only avoid the waste of network bandwidth resources but also meet the requirements of privacy and security. [7].

\subsection{Comparison of Edge Computing and Cloud Computing.}

Edge computing is not to replace cloud computing but to complement and expand cloud computing. Through edge computing, it can make up for the shortcomings of cloud computing, such as location awareness and mobile support [8]. From the perspective of bionics, edge computing and cloud computing are similar to the relationship between nerve endings and the brain. The preprocessing of data by edge computing is equivalent to the unconditioned reflex of nerve endings to external stimuli, and the historical analysis and mining of data by cloud computing are equivalent to the memory and logic processing functions of the brain. Table 1 illustrates the differences between cloud computing and edge computing in terms of computing mode and distribution characteristics [9].

\subsection{Digital Community Management. Digital information} has the advantages of no distortion, anti-interference, encryption, and easy storage. Digital management of urban communities is the introduction of information technology, especially digital technology, into community management. On the basis of the digital community system and digital management technology that has been formed, the wishes of various departments, units, and residents participating in community management are carried out. Their actions and interactions are unified and coordinated, the enthusiasm and creativity of various subjects participating in community governance are maximized and work together to do a good job in community services, the quality of community management is improved, good community governance and social order are achieved, and the needs of residents for a better life and enhance happiness are further met [10].

From the content point of view, the digital management of urban communities covers the use of digital technology in the political, economic, and cultural management of urban communities, including the establishment of community organizations, population management, education services, ecological environment governance, scientific and technological cultural services, ideological and moral construction, implementation of digital management in labor and social security, public security management, and convenience services. The foundation of digital management is to establish a digital management system, and the key to digital management is to improve various management systems and mechanisms $[11,12]$. At present, the basic digital management technology system and database that need to be established for the digital management of urban communities in my country mainly include the following four aspects:

(1) Community basic data statistics system: this system should include community area, infrastructure (amount of facilities, grade, distribution, development level, etc.), location, economic status, and population (occupation structure, age structure, gender structure, knowledge structure, etc.)

(2) Community information inquiry and help system: this system responds to various policies, regulations, and rules related to open government affairs, such as social security, military support, medical, health, and old-age care, tourism, meteorology, shopping, food and entertainment, and housekeeping, family education services, home appliance repair, job introduction, and life service information, as well as emergency services such as fire alarm, public security, ambulance, and house emergency repairs, providing inquiries and online processing services [13]

(3) Social public facilities operation system: this system is a comprehensive digital management system including road traffic, water supply, gas supply, power 


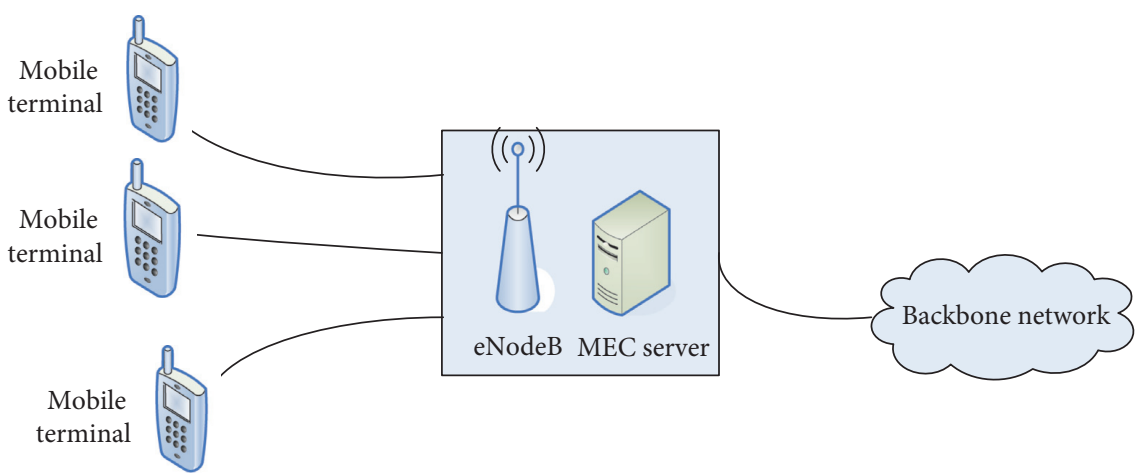

FIgURE 1: MEC architecture.

Table 1: The difference between edge computing and cloud computing.

\begin{tabular}{lcc}
\hline Types & Edge computing & Cloud computing \\
\hline Calculation mode & Distributed & Centralized \\
Distribution characteristics & Network edge & Network center \\
Service type & Lightweight small tasks & Batch processing large tasks \\
Bandwidth requirement & Not sensitive & Sensitive \\
Mobile support & Yes & No \\
Location awareness & Yes & No \\
Time delay & Low & High \\
Hardware resources & Restricted & Adequate \\
\hline
\end{tabular}

supply, communication, and other public facilities in the community to provide the community public with payment, inquiries, and inquiries that are not restricted by time and space [14]

(4) Management decision analysis system: this system is oriented to community management departments, including the analysis and prediction of community operation status, needs, problems, and benefits. It provides a decision-making basis and plans for community management departments to facilitate their timely understanding of community information, grasp the situation, and efficiently make decisions and management related to the community [15]

2.5. Trust Evaluation. The edge computing environment is essentially an open system. The trust relationship in it involves interaction, expectations, and environment. It is difficult to carry out systematic and accurate trust management. In addition to the distributed characteristics, the integration and integration of trust management are difficult. Timely feedback cannot guarantee the timeliness, and the result will lose value. In recent years, research on trust prediction models for open environments has focused on reflecting the complexity and time-varying nature of dynamic predictions, such as point-to-point computing and wireless ad hoc networks. These studies can be used as a reference for establishing a trust model for edge computing, but some issues still need to be further considered in the process of conclusion migration:
(1) The goal of trust prediction in most open environments is the overall credibility, while the existing prediction models have a large subjective component, which affects the reference value of the results, and the parameter weights of each part of the model are relatively fixed. In practical applications, the model lacks flexibility and adaptability

(2) On the basis of openness, edge computing also has the characteristics of the distribution. Some environments have requirements for individual terminal devices and overall trust value. In the process of trust feedback, the range and number of model prediction objects will directly affect the operating speed of the system and the cost of the network; that is, the size of the model processing problem is directly related to the scalability of the system

(3) The most important problem of the trust prediction model is how to ensure the timeliness of prediction results. Due to the dynamic changes of the environment, the predicted results at the current stage will gradually lose their utility over time; that is, the length of the interval directly affects the contribution of the predicted results. At present, most studies have insufficiently considered the relationship between the dynamic changes of the environment and the timeliness of the prediction results, which in turn affects the practical application value of the model [16]

In the process of performing specific computing tasks, resource constraints usually lead to huge differences in the number of AEU service requests and responses. Some AEUs 
refuse to disclose identity information due to privacy considerations but still seek interaction. Some AEUs require high-level services due to real-time services. As a security mechanism, some AEUs only hope to obtain positive benefits from resource interaction without contributing any resources they hold. The above-mentioned AEUs all have nontrust characteristics, which makes it difficult to establish a targeted trust evaluation method. In addition, it is unreliable to use a single factor to evaluate trust. For example, the authentication mechanism usually only has two results: pass or fail [17]. Considering the complexity of AEU when performing resource interaction or application services, trust values with multiple key attributes need to be considered [18].

2.5.1. Attribute-Based Trust Evaluation. There are a variety of attributes that can be used to describe terminal equipment and basic characteristics such as identity, function, data type, and area attribution, in addition to computing resources, storage size, and occupied bandwidth. First, we define the trust property of ECU as follows:

$$
V_{t}=\left\langle V_{i}, V_{b}, V_{c}>\right.\text {. }
$$

(1) Identity Trust. Identity data is the most basic data of a terminal device, so we first consider the influence of the device's identity on trust evaluation. Existing identity security management mostly relies on real-name authorization and only provides services for authorized devices. On the other hand, terminal devices have a great demand for anonymous access services. Public key infrastructure (PKI) can realize anonymous $\mathrm{P} 2 \mathrm{P}$ trusted communication in distributed networks and has been extensively studied in the identity information management of edge computing. Considering the fact that identity trust is a part of the entire trust evaluation, and some of the contents are simplified, a lightweight key generation method is proposed $[19,20]$. In the process of the resource request, the identity trust of ECU is defined as Vi. The calculation method of $\mathrm{Vi}$ is realized by the elliptic curve encryption algorithm ECC. First, a discrete elliptic curve $E$ is given by the ECC algorithm as shown in

$$
E: y^{2}=x^{3}+a x+b(\bmod \mathrm{p}) \text {. }
$$

First, we randomly select a point $O(x, y)$ on the curve and then generate the public key and private key through the following two steps:

Step 1: server key generation edge server generates a key pair $\left(K_{p}, K_{s}\right)$ according to the following formulae:

$$
\begin{gathered}
K_{p}=H\left(I_{l}, I_{e}\right), \\
K_{s}=M\left(K_{p}\right) .
\end{gathered}
$$

The formula $K_{p}$ is obtained by the hash function $\mathrm{H}$ about the ECU identity information $I_{l}$ and the edge service information $I_{e}$. After obtaining the public key $K_{p}$, the private key is obtained through the encoding function $M$ on $K_{p}$. After calculating the key, the edge server sends the identity information to the ECU, and the identity information is shown in

$$
D=\left\{K_{p}, E, O\right\} .
$$

Step 2 (ECU key generation): after the ECU receives the identity information $D$, it can generate three key formulae:

$$
\begin{aligned}
& K_{v i}=R_{1}(O(x, y)), \\
& K_{e p}=R_{2}(O(x, y)), \\
& K_{e s}=R_{1}\left(M\left(K_{e p}\right)\right) .
\end{aligned}
$$

The virtual key $K_{v i}$ is used to protect the real identity information of the ECU, $K_{e p}$ and $K_{e s}$, respectively, represent the public key and private key generated by the ECU, and $R 1$ and $R 2$ are pseudorandom number generation functions.

Then, the ECU will send the information $F$ to the edge server for key matching, and $\alpha$ represents the temporary parameter. The expression of information $F$ is shown in

$$
F=\left\{K_{v i}, K_{e p}, \alpha\right\} .
$$

(2) Behavioral Trust. The terminal equipment sending out service requests or responses is an important basis for trust evaluation. Behavior involves many factors. Because the edge computing environment requires real time and effectiveness, and terminal equipment usually has limited resources, trust evaluation is being carried out. At this time, it is necessary to focus on factors directly related to trust evaluation. The behavioral credibility shown in the service request or response process is defined as behavioral trust $\mathrm{Vb}$, which is mainly composed of three parts in

$$
V_{b}=w_{b v} V_{b c}+w_{b e} V_{b e}+w_{b r} V_{b r} .
$$

(3) Behavior Constraints. Behavioral constraints Vbc represent the specifications that the terminal must comply with when running in the current environment, including interaction constraints or interaction specifications for specific behaviors, such as minimum duration and moving range. Generally, the terminal is subject to several constraints [21]. When the specific calculation tasks of the ECU change, the corresponding specifications usually change. Vbc can be expressed by

$$
V_{b c}=w_{1} \xi_{1}+w_{2} \xi_{2}+\cdots+w_{c} \xi_{c} .
$$

(4) Behavioral Interaction. As the edge computing environment continues to change, it is necessary to consider the impact of changes in terminal behavior on trust evaluation. This paper adopts the method of time series analysis to avoid the limitation of terminal type for trust evaluation, analyze the behavior changes of other terminal devices and the device during the interaction, and reduce the computational burden caused by trust analysis. First of all, the behavioral interaction of the target ECU is defined as Vbe. Vbe reflects the credibility of the interaction in the past 
period of time. The most basic interaction can also get an effective trust evaluation. If the ECU has not interacted before, the default Vbe is 0.5 ; the time series can be defined as follows:

$$
S_{j}(\Delta t)=\left\{\tau_{j}^{1}, \tau_{j}^{2}, \ldots \tau_{j}^{s} \ldots \tau_{j}^{t}\right\} .
$$

For $\mathrm{ECU}_{j}$, a temporary threshold $\alpha_{j} \in(0,1)$ is allocated to it according to the total amount of interactive resources, which is defined as follows:

$$
H_{j}^{+}(\Delta t)=\sum_{i=1}^{t} 1\left(\tau_{j}^{i} \geq \alpha j\right)
$$

Vbe can be derived from

$$
V_{b e}=\frac{1}{k} \sum_{j=1}^{k} \frac{H_{j}^{+}(\Delta t)}{t}
$$

(5) Behavior Change Rate. The change rate of terminal behavior usually means the change of trust. It is necessary to study whether there is a risk in this change. To define the change rate of behavior as $\mathrm{Vbr}$, then there is

$$
V_{b r}=\frac{1}{k} \sum_{j=1}^{k}\left(\frac{1-\lambda V_{b e \Delta(t-1)}}{1+\sqrt{t-H_{j}^{+}(\Delta(t-1))}}+\frac{\lambda V_{b e \Delta(t)}}{1+\sqrt{H_{j}^{+}(\Delta t)}}\right) .
$$

\section{Digital Community Management Mobile Information System Based on Edge Computing}

3.1. Overall Architecture of the Community Management Mobile Information System. In the current system development stage, some large-scale software systems are programmed to develop using object-oriented development methods. The object-oriented method is mainly derived from specific software-oriented programming, and its fundamental meaning is to target specific software. We purposely develop some software systems in the things around us and express our human thoughts and thinking as much as possible in this system software. The development of application software is mainly aimed at people's living habits and characteristics. A large part of subjectively developed programs that conform to people's lifestyles is aimed at some special masses. For the community users themselves, targeted development programs are for more systematic use of the management operating system, making both users and managers have a certain degree of practicality and convenience [22]. Figure 2 is the overall architecture of the development system.

Community managers can $\log$ in to this system to manage the information of the residents of the community and update the information of house rental and sale in real time and can also add, delete, modify, and check the local database.
3.2. System Design Principles. The design of a digital community management mobile information system based on edge computing is a very complex project. In the design, the following five aspects are mainly considered:

(1) The practicality of the system: to be simple, practical, and humanized, to achieve a unified application interface, unified user management, and unified identity authentication, and to meet the needs of personalized information services [23]

(2) Advancement: it should be advanced and forwardlooking in design ideas, system structure, and platform selection; it adopts advanced and mature technologies to meet the current needs of community management, take into account other related management needs, and maintain technological advancement for a period of time [24]

(3) Openness: the system structure, technology adopted, and platform selection should all be standard and open. It can exist independently and coexist with other systems, and it can also support subsequent development and research

(4) Reliability: the system design must meet the design requirements of performance indicators. The network structure, network equipment, server equipment, etc. must be designed and constructed with high reliability to improve the safety and reliability of the entire digital community computer network and application system

(5) Scalability: the construction of the digital community needs to be gradually and continuously expanded. For this reason, the system needs to adopt component technology to support the combination, inheritance, and online definition of applications [25]

3.3. Module Function Design of the System. After analyzing the needs of the platform, the platform is finally designed into 3 functional modules, including the community information query module, community service module, and fee payment module. The detailed module framework of the system is shown in Figure 3.

The community information query module can help community residents keep abreast of real-time news reports and community announcements. The community service module can help residents understand the latest employment trends and information about housing rentals and sales. The fee payment module can make it more convenient and fast for owners to pay for utilities and other living expenses.

3.4. Main Database Design. The functions of the community digital management mobile information network system are very comprehensive, so there are many tables involved in the system, and it is not possible to list them all. Here, only the structure of the tables involved in the implementation of the system is listed as follows. The user information table is 


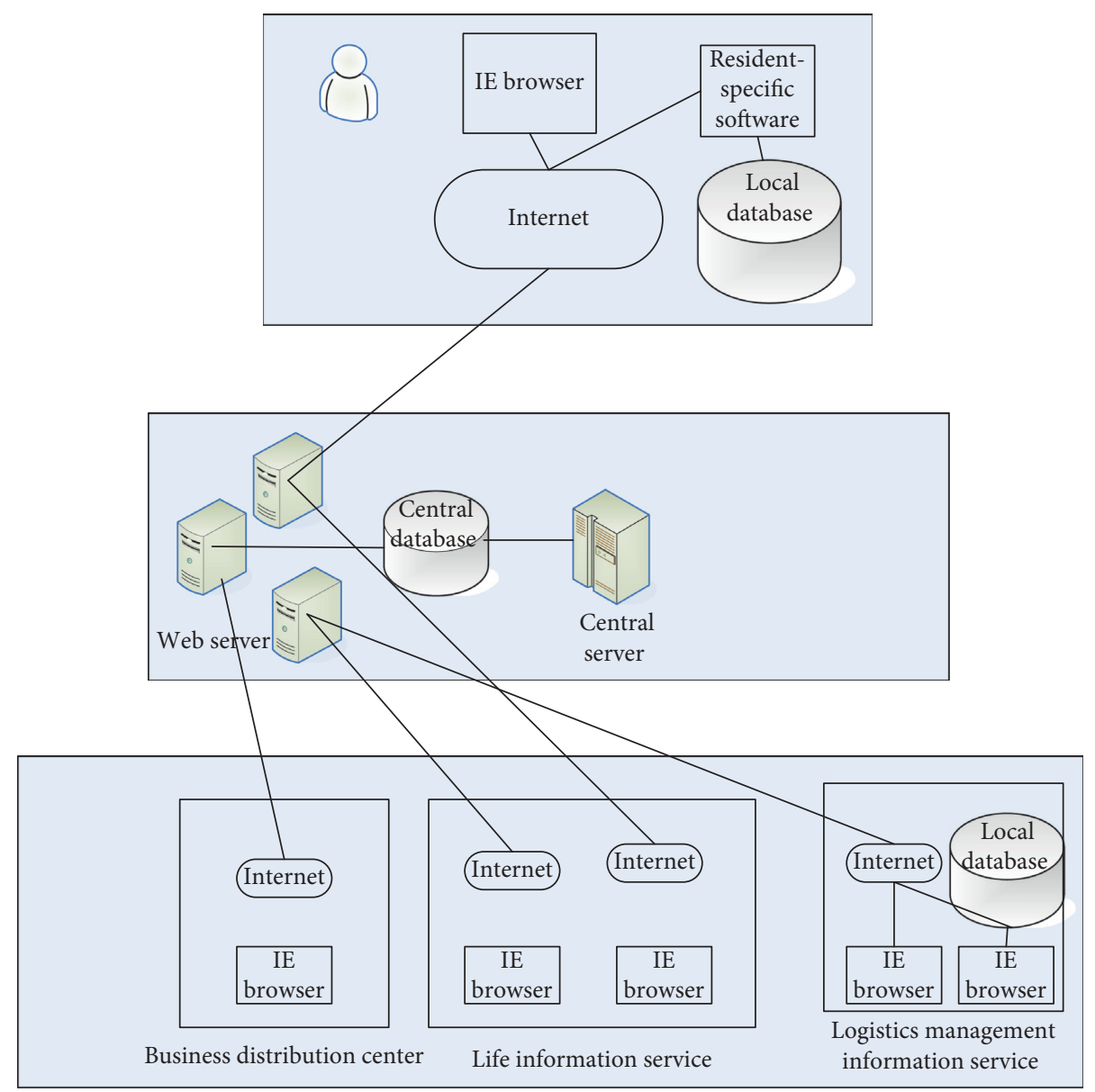

FIgURE 2: The overall architecture of the system.

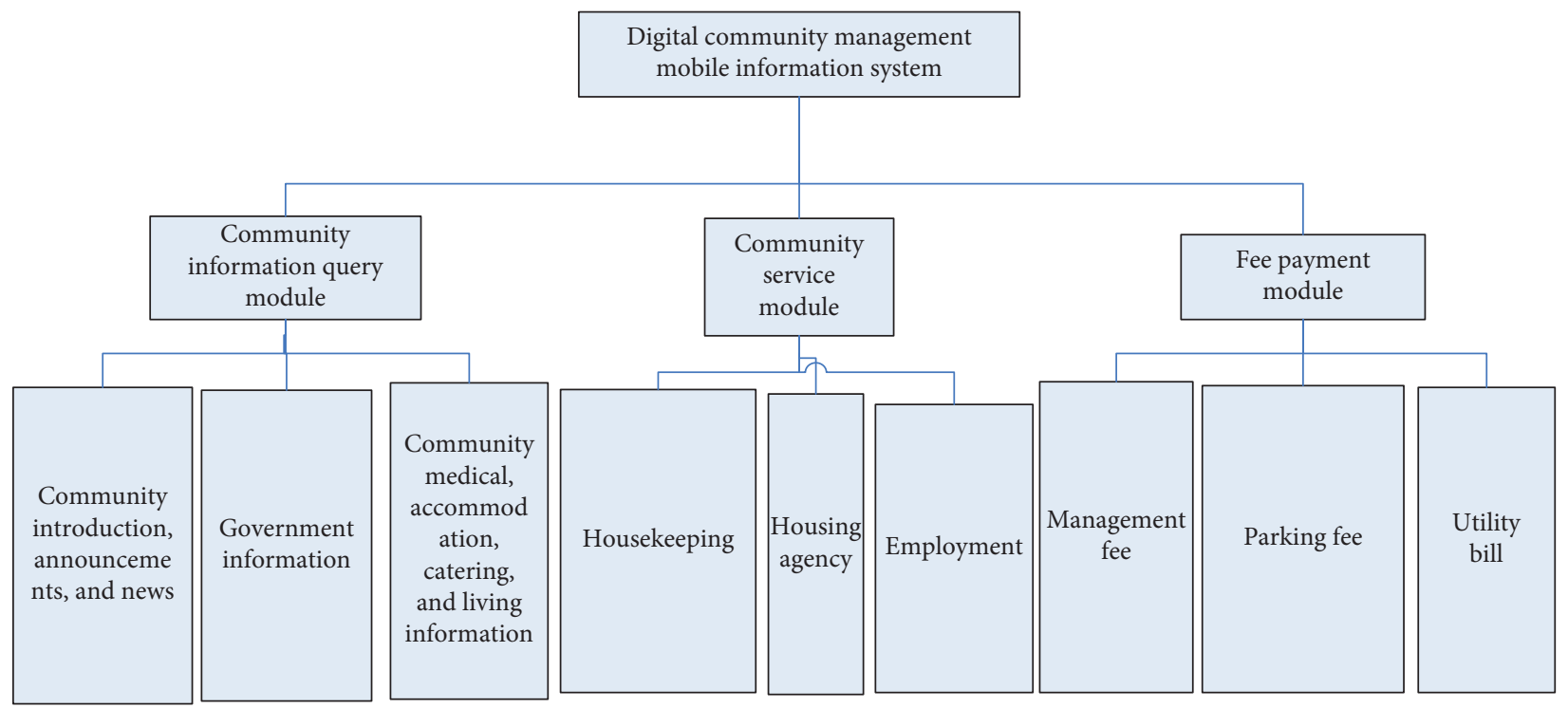

FIgURE 3: Frame diagram of each module of the system.

mainly used to store the basic information of the unit or user, the unit user can register its legal person information, and the household can register the head of the household information. As the basis for information release, the registration information must be true and reliable. Among them, ID is the user number, which is an automatically increasing field and does not need to be filled in during registration. The user name is the nickname registered by the 
user, which can use any characters, the password is at least six characters, and the rest of the personal information is filled in according to actual conditions. Its structure is shown in Table 2.

The community information query table is mainly used to store various dynamic information in the information query module. The ID field is an autogrowth field, and it is not necessary to fill in when adding records to the table. The information type field can be government affairs information, community announcements, system specifications, community news, life information, etc., with information numbers 1-12 assigned. The title of the information is in characters and its length does not exceed 50. When the information exceeds 50 , the overlength part will be ignored. The publishing department field is used to record the publishing unit or individual of information, and the content is text type. You can use copy and paste operations to fill in. The management of the content in the News table is done by the system administrator, and various information can be added, modified, and deleted in the community information management submodule of the system management module. Its structure is shown in Table 3.

The real estate information table is used to save real estate information released by users and also as a data source for querying real estate information. The ID field is an automatic growth field, which is filled in by the system. The information category is rent or sale, represented by 0 or 1 . Other real estate-related pieces of information are filled according to the actual situation. The release date is automatically filled in by the system. The validity period is the default time limit of the system. If it needs to be changed, the system administrator can modify it in the housing intermediary information management submodule of the department management module. The user name field registered by the publisher for the user who logs in to the system to publish information is a nickname. The management of the information in the real estate information table, such as modification and deletion of expired information, is performed by the system administrator in the housing intermediary information management submodule of the department management module. Its structure is shown in Table 4.

The expense payment table is used to save the owner's various living expenses information. As long as the user has registered in the community, a record will be generated in the fee payment table, and the user can apply for a service agency to pay certain fees. Among them, ID is the unique number assigned by the system when the user is registered. By associating with the User table, the user's nickname, real name, community account, and account balance can be obtained. The name of the fee can be property fees, management fees, parking fees, orders, water, electricity and gas bills, telephone fees, mobile phone fees, Internet fees, and other fees incurred by transactions in the flea market. Its structure is shown in Table 5.

The user message table is mainly used in the community message module in other business modules to save the user's message information. The ID field is an autogrowth field filled in by the system, the message user ID is the ID number
TABLE 2: User information table.

\begin{tabular}{lcc}
\hline Field name & Types & Length \\
\hline Id & Int & 4 \\
UserName & Vchar & 20 \\
Passward & Vchar & 20 \\
RealName & Vchar & 20 \\
Tel & Vchar & 20 \\
ID card & Vchar & 18 \\
Post & Vchar & 6 \\
\hline
\end{tabular}

TABLE 3: Community information query form.

\begin{tabular}{lcc}
\hline Field name & Types & Length \\
\hline ID & Int & 4 \\
NewsType & Int & 4 \\
NewsTitle & Vchar & 50 \\
PublishDep & Vchar & 20 \\
PublishDate & Vchar & 16 \\
NewsContent & Text & - \\
\hline
\end{tabular}

TABle 4: Property information sheet.

\begin{tabular}{lcc}
\hline Field name & Types & Length \\
\hline ID & Int & 4 \\
Infype & Int & 1 \\
BedRoom & Int & 1 \\
Hall & Int & 1 \\
WashRoom & Int & 1 \\
Acreage & Int & 1 \\
Floor & Int & 1 \\
Age & Vchar & 16 \\
\hline
\end{tabular}

TABle 5: Fee payment table.

\begin{tabular}{lcc}
\hline Field name & Types & Length \\
\hline ID & Int & 8 \\
ExpenseName & VChar & 20 \\
Period & VChar & 50 \\
Price & Int & 4 \\
EndDate & Datatime & 16 \\
CommissionCharge & Int & 4 \\
Note & Text & - \\
\hline
\end{tabular}

when the user registered, the message user name is the user nickname, the message title and message content are filled in by the user, and the message time is automatically filled in by the system according to the current date and time. The maintenance of the table is performed by the system administrator in the community message management submodule of the system management module and can mark user messages or delete expired messages. Its structure is shown in Table 6.

\section{System Performance Analysis}

4.1. System Simulation Experiment Analysis. After completing the overall design of the experiment and the analysis 
TABle 6: User message form.

\begin{tabular}{lcc}
\hline Field name & Types & Length \\
\hline ID & Int & 8 \\
userId & Int & 8 \\
Name & VChar & 20 \\
Title & Varchar & 100 \\
Body & Text & - \\
UpdateTime & Datatime & - \\
\hline
\end{tabular}

TABLE 7: System development environment and development tools.

\begin{tabular}{lc}
\hline CPU & Intel Core i7-7300u \\
\hline GPU & GeForce GTX 1050Ti \\
Operating system & Windows 10 professional edition 64 bit \\
RAM & $16 \mathrm{~GB}$ \\
Jre version & 1.7 \\
Development platform & IDEA \\
Server & Tomcat8.3 \\
\hline
\end{tabular}

of functional module requirements, we conducted simulation experiments on the digital community management mobile information system based on edge computing in the next few months. We invited 102 community owners from a certain community to adopt. The community management system based on edge computing designed in this paper was tested. The development environment and development tools are shown in Table 7.

We conducted a multifaceted questionnaire survey on these 102 community owners. A total of 102 questionnaires were issued, 101 questionnaires were returned, and 1 invalid questionnaire was eliminated. The remaining 100 valid questionnaires were statistically explained. The result is shown in Figure 4, A represents that residents are more satisfied with the payment method, $B$ represents more standardized community management, and $C$ represents more timely news.

It can be seen from Figure 4 that most of the owners believe that the system design has made community management more standardized, and more than $40 \%$ of the owners believe that the system design has made it easier and faster for them to pay for utility bills, especially when they are older. Small owners agree more with this system design.

4.2. Satisfaction Survey of the Mobile Information System. In addition, we also conducted a satisfaction survey on the mobile information system designed this time; the 100 community owners who participated in the satisfaction survey have the same comprehensive situation in terms of moral quality and physical and mental health, etc. And the survey results are shown in Figure 5:

It can be seen from Figure 5 that most of the young owners are satisfied with the system design. They are more familiar with the operation of the community management system. There is a large majority of the elderly who are not satisfied with the management system designed this time. It is not humane enough, and there are no operating prompts for the elderly. It is easy to misunderstand the elderly. I hope

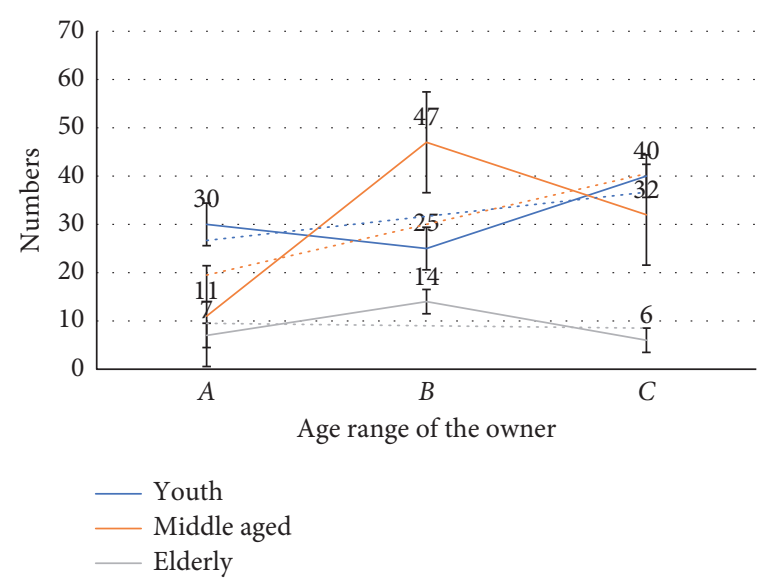

Figure 4: Questionnaire for owners of different ages.

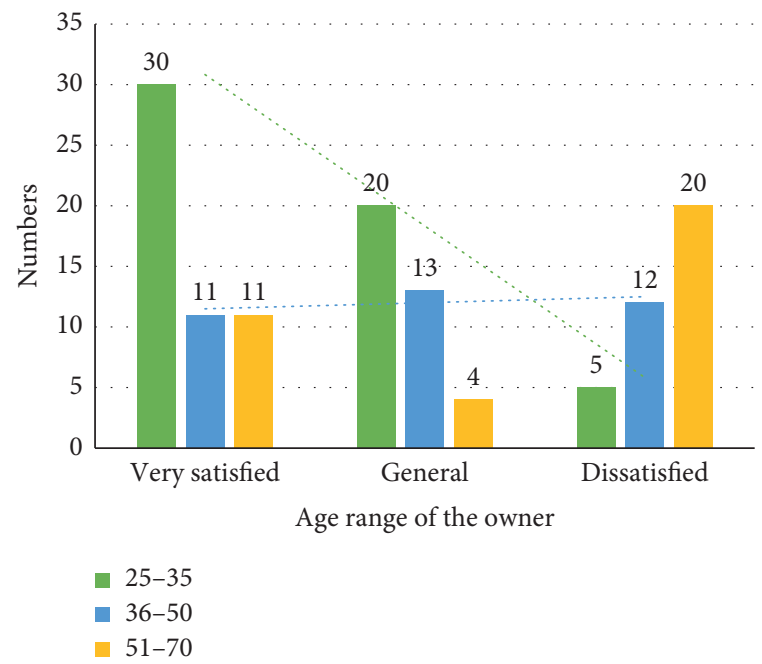

FIGURE 5: Satisfaction degree of owners of different age groups with the system.

that the management system designed this time needs to be improved and perfected.

\section{Conclusions}

The digital community management mobile information system designed and developed in this paper based on edge computing has brought convenience to our lives. However, due to the tight development time, this system still has many shortcomings that need to be improved and there are many module functions that have not been implemented. The construction of a digital community management mobile information system based on edge computing in my country is still in its infancy. The wishes of various departments, units, and residents are scattered and cannot be effectively unified. It is necessary to achieve a ladder from the initial stage, the intermediate stage to the advanced stage. For development, there are still a lot of problems and difficulties that need to be solved. On the one hand, we must break down the barriers between departments, effectively coordinate the actions and interactions of various units and 
departments in the jurisdiction, mobilize the enthusiasm of all entities involved in community management to the greatest extent, give play to their respective creativity, and work together to do a good job in the community. We provide services to improve the quality of community management and achieve a good new order of community governance. On the other hand, it is necessary to standardize various technical standards, provide digital languages that can be shared, establish a perfect exchange platform, continuously repair and complete various management systems and mechanisms, and do a good job in the construction of the basic database framework of urban communities, which is a basic database statistics system for communities. Information inquiry help system, social public facilities operation system, and management decision analysis system provide operation platform and technical support.

\section{Data Availability}

No data were used to support this study.

\section{Conflicts of Interest}

The author declares no conflicts of interest in our paper.

\section{Authors' Contributions}

The author read the manuscript and approved to submit it to the journal.

\section{Acknowledgments}

This paper was funded by the Zhejiang Civil Affairs Policy Theoretical Research Planning Project: "Research on the PATH of Intelligent Improvement of Grassroots Community Governance" (No. ZMKT202019).

\section{References}

[1] Z. Xiong, Y. Zhang, N. Dusit et al., "When mobile blockchain meets edge computing," IEEE Communications Magazine, vol. 56, no. 8, pp. 33-39, 2017.

[2] T. Taleb, K. Samdanis, B. Mada, H. Flinck, S. Dutta, and D. Sabella, "On multi-access edge computing: a survey of the emerging 5G network edge cloud architecture and orchestration," IEEE Communications Surveys \& Tutorials, vol. 19, no. 3, pp. 1657-1681, 2017.

[3] P. Hutchings, R. Franceys, S. Jasthi et al., "Community management and participation in multi-village schemes for rural water supply in India," Waterlines, vol. 39, no. 2-3, pp. 113-143, 2020.

[4] P. Corcoran and S. K. Datta, "Mobile-edge computing and the internet of things for consumers: extending cloud computing and services to the edge of the network," IEEE Consumer Electronics Magazine, vol. 5, no. 4, pp. 73-74, 2016.

[5] S. Pan, L. Zheng, X. Zheng et al., "Application of five-in-one community management mode plus Wuhan's regional WeChat platform in the novel coronavirus control," Open Journal of Nursing, vol. 10, no. 3, pp. 252-259, 2020.

[6] B. P. Rimal, D. P. Van, and M. Maier, "Mobile-edge computing empowered fiber-wireless access networks in the $5 \mathrm{G}$ era," IEEE Communications Magazine, vol. 11, no. 2, pp. 192-200, 2016.

[7] M. B. Hasan, P. P. J. Driessen, S. Majumder, A. Zoomers, and F. Van Laerhoven, "A community management plus model for the governance of rural drinking water systems: a comparative case study of pond sand filter systems in Bangladesh," International Journal of the Commons, vol. 14, no. 1, pp. 662-679, 2020.

[8] X. Chen, Q. Shi, L. Yang, and J. Xu, "ThriftyEdge: resourceefficient edge computing for intelligent IoT applications," IEEE Network, vol. 32, no. 1, pp. 61-65, 2018.

[9] E. Ahmed, A. Ahmed, I. Yaqoob et al., "Bringing computation closer toward the user network: is edge computing the solution?" IEEE Communications Magazine, vol. 55, no. 11, pp. 138-144, 2017.

[10] K. Kaur, S. Garg, G. S. Aujla, N. Kumar, J. J. P. C. Rodrigues, and M. Guizani, "Edge computing in the industrial internet of things environment: software-defined-networks-based edgecloud interplay," IEEE Communications Magazine, vol. 56, no. 2, pp. 44-51, 2018.

[11] L. Zhi, W. M. Wang, L. Guo et al., "Toward open manufacturing: a cross-enterprises knowledge and services exchange framework based on blockchain and edge computing," Industrial Management \& Data Systems, vol. 118, no. 9, pp. 303-320, 2018.

[12] R. Wang, J. Yan, D. Wu, H. Wang, and Q. Yang, "Knowledgecentric edge computing based on virtualized D2D communication systems," IEEE Communications Magazine, vol. 56, no. 5, pp. 32-38, 2018.

[13] J. Liu, J. Dong, H. Long, T. Xu, and L. Putzel, "Private vs. community management responses to de-collectivization: illustrative cases from China," International Journal of the Commons, vol. 14, no. 1, pp. 445-464, 2020.

[14] M. Satyanarayanan, "The emergence of edge computing," Computer, vol. 50, no. 1, pp. 30-39, 2017.

[15] D. Lunzhi, "Anonymous certificateless multi-receiver encryption scheme for smart community management systems," Soft Computing, vol. 24, no. 1, pp. 281-292, 2020.

[16] S. Nastic, T. Rausch, O. Scekic et al., "A serverless real-time data analytics platform for edge computing," IEEE Internet Computing, vol. 21, no. 4, pp. 64-71, 2017.

[17] A. Hadiana and A. Ginanjar, "Designing interface of mobile parental information system based on users' perception using Kansei engingeering," Journal of Data Science and Its Applications, vol. 1, no. 1, pp. 10-19, 2018.

[18] Y. Wang, S. Min, X. Wang et al., "Mobile-edge computing: partial computation offloading using dynamic voltage scaling," IEEE Transactions on Communications, vol. 64, no. 10, pp. 4268-4282, 2016.

[19] A. Gumaei, M. Al-Rakhami, M. M. Hassan et al., "Deep learning and blockchain with edge computing for 5G-enabled drone identification and flight mode detection," IEEE Network, vol. 35, no. 1, pp. 94-100, 2021.

[20] Y. Mao, J. Zhang, S. H. Song, and K. B. Letaief, "Stochastic joint radio and computational resource management for multi-user mobile-edge computing systems," IEEE Transactions on Wireless Communications, vol. 16, no. 9, pp. 5994-6009, 2017.

[21] W. Kazimierski and M. Włodarczyk-Sielicka, "Technology of spatial data geometrical simplification in maritime mobile information system for coastal waters," Polish Maritime Research, vol. 23, no. 3, pp. 3-12, 2016.

[22] W. Shi, J. Cao, Q. Zhang, Y. Li, and L. Xu, "Edge computing: vision and challenges," IEEE Internet of Things Journal, vol. 3, no. 5, pp. 637-646, 2016. 
[23] Z. Ke, Y. Mao, S. Leng et al., "Mobile-edge computing for vehicular networks: a promising network paradigm with predictive off-loading," IEEE Vehicular Technology Magazine, vol. 12, no. 2, pp. 36-44, 2017.

[24] G. Ananthanarayanan, P. Bahl, P. Bodík et al., "Real-time video analytics: the killer app for edge computing," Computer, vol. 50, no. 10, pp. 58-67, 2017.

[25] G. Liu, S. Fei, Z. Yan, C.-H. Wu, S.-B. Tsai, and J. Zhang, "An empirical study on response to online customer reviews and E-commerce sales: from the mobile information system perspective," Mobile Information Systems, vol. 2020, no. 83, pp. 1-12, 2020. 